\section{P24 Frequency of Eating Alone Among Adolescents was Associated with Parent Support, Dietary Intake and BMI: FLASHE Study Results}

Marla Reicks,PhD, RD, mreicks@umn.edu, University of Minnesota, 1334 Eckles Avenue, St. Paul, MN 55018;

Cynthia Davey, MS; Alex Kojo Anderson, PhD, MPH, University of Georgia; Jinan Banna, PhD, RD, CDN, University of Hawaii; Mary Cluskey, $P h D, R D$, Oregon State University; Carolyn Gunther, PhD, RD, Ohio State University; Blake Jones, PhD, Purdue University; Rickelle Richards, PhD, Brigham Young University; Glade Topham, PhD, Kansas State University; Siew Sun Wong, PhD, Oregon State University

Background (Background, Rationale, Prior Research, and/or Theory): Often eating alone among adolescents may negatively affect dietary intake and weight status because of limited perceived parental support for healthy eating.

Objective: To examine associations between frequency of adolescents' eating alone, dietary behaviors, perceived parental support, and weight status.

Study Design, Setting, Participants, Intervention: The Family Life, Activity, Sun, Health, and Eating (FLASHE) Study collected data on food behaviors and environmental factors from a nationwide sample of parent-adolescent (12-17 years) dyads in 2014 via a cross-sectional web-based survey using a consumer opinion panel. Agreement with "In my family: I often eat alone" was dichotomized as somewhat/strongly agree $(n=343)$ (often eating alone) versus neither/ somewhat/strongly disagree $(n=1,309)$ (not often eating alone).

Outcome Measures and Analysis: Chi-square/twosample t-tests and logistic regression analysis were used to test for associations between variables.

Results: Adolescents often eating alone were older and more likely to be overweight/obese than those not often eating alone. Among those often eating alone compared to those not often eating alone, the frequency was higher for the evening meal being delivered or made from a box meal, mean daily intake frequency was higher for junk food (JF) and sugary drinks and lower for fruit and vegetables (FV), and fewer agreed that their parents support healthy FV behaviors and limit JF and sugary drinks. Adjusted odds of adolescents often eating alone were significantly lower for those who reported that FV were often/always available in the home $(\mathrm{OR}=0.67)$, for those who agreed that they made decisions with parents about how many FV they have to eat $(\mathrm{OR}=0.69)$, and for those who agreed that it's OK for parents to make rules about the quantity of JF/sugary drinks they can have $(\mathrm{OR}=0.72)$.

Conclusions and Implications: Often eating alone was related to higher BMI, less healthy dietary intake, and lower perceived parental support compared to not often eating alone indicating a need for parental strategies to support adolescent healthy eating choices when eating alone.

Funding: None.

\section{P25 Gender Comparisons in Young Adult Eating Behavior Regulation}

Tessa Hamilton, EdM, CAS, hamilton.t@husky.neu.edu, Northeastern University,

426 International Village, 360 Huntington Avenue, Boston, MA 02115; Jessica Hoffman, PhD, Northeastern University; Dilbur Arsiwalla, PhD, University of Northern Iowa; Robert Volpe, PhD, Northeastern University; Ellyn Schmidt, MS; Sareen Gropper, PhD, RD, LD, Florida Atlantic University

Background (Background, Rationale, Prior Research, and/or Theory): The contextual assessment of motivation to regulate one's eating behaviors can enhance our understanding of why some individuals are successful at managing their consumption patterns while others may continue to struggle (Pelletier, Dion, Slovenic-D'Angelo, \& Reid, 2004). Prior studies have demonstrated that intrinsic motivation is associated with factors protective against obesity and are less likely to be disordered than externally motivated eating behaviors (Pelletier \& Dion, 2007; Pelletier et al., 2004; Pelletier, Dion, \& Levesque, 2004). However, eating behavior regulation may occur differently across genders; women are more likely than men to engage in eating regulation in an effort to improve health, attach a higher level of importance to healthy eating, and to view improving health as a motivator for modifying eating behaviors (Wardle et al., 2004).

Objective: The objective of this study was to evaluate how men and women differed in their motivation to engage in eating behavior regulation.

Study Design, Setting, Participants, Intervention: This was assessed through the administration of the Regulation of Eating Behavior Scale (Pelletier et al., 2004), a measure designed to assess dietary behavior regulation according to styles proposed by self-determination theory (SDT; Deci \& Ryan, 1985) in a sample of 536 undergraduate students in the southeastern United States. A multivariate analysis of variance (MANOVA) was used to examine gender differences.

Conclusions and Implications: A significant main effect was detected for gender across the six subscales, F(6, $491)=10.56, P<.001$, Pillai's Trace $=.11$, Partial $\eta 2=.11$ ). Subscale score differences were statistically significant between men and women on all but two types of eating regulation, suggesting that men and women in the sample experienced motivation to engage in eating behavior regulation differently. Thus, educational programs and intervention programs may need to be differentiated for men and women to account for these motivational differences.

Funding: None. 